\title{
Toward the control of the creation of mixed monolayers on glassy carbon surfaces by amine oxidation.
}

\author{
Jessica Groppi, ${ }^{a}$ Philip N. Bartlett ${ }^{\mathrm{b}}$ and Jeremy D. Kilburn ${ }^{\mathrm{c}}$ \\ ${ }^{a}$ Jessica Groppi: School of Biological and Chemical Sciences, Queen Mary University of London, Mile \\ End Road, London, E1 4NS (UK). E-mail: j.groppi@qmul.ac.uk \\ b Prof. Philip N. Bartlett: Chemistry, Faculty of Natural and Environmental Sciences, University of \\ Southampton, Southampton, SO17 1BJ (UK). E-mail: P.N.Bartlett@ soton.ac.uk \\ ${ }^{c}$ Prof. Jeremy D. Kilburn: King's College, The University of Aberdeen, Aberdeen, AB24 3FX (UK). \\ E-mail: j.kilburn@abdn.ac.uk
}

\begin{abstract}
A versatile and simple methodology for the creation of mixed monolayers on glassy carbon (GC) surfaces was developed, using an osmium bipyridyl complex and anthraquinone as model redox probes. The work consisted in the electrochemical grafting on GC of a mixture of mono-protected diamine linkers in varying ratios which, after attachment to the surface, allowed orthogonal deprotection. After optimisation of the deprotection conditions, it was possible to remove one of the protecting groups selectively, couple a suitable osmium complex and cap the residual free amines. The removal of the second protecting group allowed the coupling of anthraquinone. The characterisation of the resulting surfaces by cyclic voltammetry showed the variation of the surface coverage of the two redox centres in relation to the initial ratio of the linking amine in solution.
\end{abstract}

\section{Introduction}

The covalent modification of electrode surfaces has been one of the central aspects in the development of electrochemical devices for the last forty years, since it represents a powerful tool for improving the stability and lends new properties to the modifed surfaces. Extensive reviews are available, describing the methodologies for the creation of organic layers covalently bonded to various electrode materials. ${ }^{1}$ Amongst these techniques, the electrooxidation of primary diamines at glassy carbon (GC) surfaces has received considerable attention in recent years ${ }^{2}$ and, applied in conjunction with solid-phase synthesis techniques, allows the linking of different redox centres to the electrodes. ${ }^{3}$ A step forward in the modification of electrodes is represented by the creation of 
mixed monolayers. The development of methodologies for the creation of mixed monolayers has been the focus of research in various fields, from the creation of semiconductors to organic electronics and sensors. ${ }^{4}$ The appeal of mixed monolayers resides in the possibility of assembling multiple components in a bottom-up approach, with control over their organisation to the molecular level. ${ }^{5}$ Currently most of the studies focus on mixed SAMs on gold followed by the electrografting of aryl diazonium salts.

The production of mixed SAMs has been achieved through several techniques: electrochemical oxidation of thiols followed by a replacement reaction ${ }^{6}$ or selective reduction of adsorbed thiols ${ }^{7}$ and electrochemical control, ${ }^{8}$ although binary monolayers of thiols are commonly obtained by coadsorption. ${ }^{9}$ Usually, the purpose of mixed SAMs is the creation of surfaces where a linker is surrounded by a diluting compound. Gooding et al. published a series of papers describing mixed SAMs in which ferrocene or anthraquinone derived norbornylogous bridges along with alkyl chains of different length as diluents, were used to study the behaviour of redox probes within the electrical double layer. ${ }^{10}$ Lee et al. recently reported the modification of gold surfaces with mixed SAMs of 1-undecanethiol and 2-bromoisobutylate terminated undecanethiol, as initiator platforms for the polymerisation of pOEGMA brushes modified with biotin for the binding of proteins. ${ }^{11}$ The use of diazonium salts extends the possibility of applying mixed monolayers to a wide range of materials, including carbon. Gooding et al. first reported the electrografting of mixtures of diazonium salts on gold in 2005, ${ }^{12}$ and since then they have studied the mechanism of grafting and the properties of the mixed layers both on gold and glassy carbon. ${ }^{13}$ They used this technique for the creation of surfaces modified with oligo(phenylethynylene) molecular wires diluted with poly(ethylene-glycol). The molecular wire provided a rigid anchoring group for the covalent attachment of horseradish peroxidase, with good electron transfer kinetics. ${ }^{14}$ Further work involved the use of mixtures of molecular wires alternated with aryl carboxylic acid groups, whose role was both to anchor and stabilise a glucose oxidase enzyme and orient the active site toward the electrode at the electrode surface. ${ }^{15}$ In both cases the direct electron transfer between enzyme and electrode was investigated. Most recently the combination molecular wires/poly(ethyleneglycol) was applied to the creation of an electrochemical immuno-biosensor. ${ }^{16}$ In separate studies, Downard et al. reported the formation of mixed monolayers by sequential electrografting of diazonium salts and the use of a bulky protecting group on the first modifier allowed enough space for the second group to attach to the surface. ${ }^{17}$

Our group reported a different approach to the creation of mixed monolayers. A monolayer of 
N-Boc-ethylenediamine was formed by oxidation of the free primary amine and removal of the protecting group. The resulting surface was modified with three different redox probes by sequentially dipping the electrode in three different coupling solutions each containing a single component. $^{18}$ Alternatively, mixed monolayers of diamines could be obtained by simultaneous electrografting of two amines in solution in varying relative ratios. ${ }^{19}$

The use of mixed monolayers with two or more components presents many advantages in the development of biosensors: enzymes can be anchored to the surface and surrounded by redox mediators to improve the mediated electron transfer and their orientation, density and distance to the surface could be regulated, leading an optimal response from the system. Moreover the approach could be used to create models that mimic the environment of the active site of enzymes by surrounding redox mediators with functionalities that could affect their physical and electrochemical properties. However, in order to create such carefully engineered surfaces it is crucial that the mixed monolayers can be created both reliably and predictably, and although it is possible to anticipate a number of practical issues that might need to be overcome in order that such reliability and predictability are achieved, to date no studies have been undertaken to examine the practical difficulties or to provide solutions for them. In this paper we describe fundamental studies directed at just such an objective and demonstrate that with careful experimental techniques predictability and reliability is achievable.

Here we report the development of a methodology to create mixed monolayers at glassy carbon surfaces in a reliable and reproducible way, using the oxidation of primary amines and solid phase synthesis as the main tools. In the model studies reported here we used two different redox mediators, an osmium bipyridyl complex and an anthraquinone, to measure the surface coverage electrochemically. These two redox groups are of significantly different size, a common situation in the design of mixed layers for particular applications, and a situation that creates its own problems as we shall see.

Osmium complexes have found wide application as redox mediators in biosensing, given the easily tunable potential of the $\mathrm{Os}^{2+/ 3+}$ couple by changing the nature of the ligands and their higher stability compared to other metal complexes. ${ }^{20}$ Moreover the steric bulk of the metal complex appears to prevent its adsorption on graphite surfaces, a process that is often unavoidable with other redox mediators such as organic dyes and quinones. Most of the literature concerning the use of osmium complexes as redox mediators involves their embedding in polymeric matrices or hydrogels, where one of the ligands is cross-linked in the polymeric backbone in order to avoid leakage of the species. ${ }^{21}$ Recently the focus has shifted to the development of methods for the 
covalent attachment of the complexes through SAMs or reduction of diazonium salts. ${ }^{22}$

\section{Results and discussion}

\section{Synthesis and characterization of bis-(2,2'-bipyridyl)(4-(pyridin-4-yl)butanoic acid)chloride osmium (II) hexafluophosphate (5)}

The synthetic pathway for osmium complex 5 is presented in Scheme 1. The precursor Os $(\mathrm{bpy})_{2} \mathrm{Cl}_{2}(\mathbf{3})$ was synthesised following a literature procedure. ${ }^{20}$ Pyridine ligand $\mathbf{1}$ functionalised with a butanoic acid moiety at $\mathrm{C} 4$ was synthesised by reacting 4-vinylpyridine with diethylmalonate, followed by hydrolysis and decarboxylation under acidic conditions. ${ }^{23}$ In order to avoid chelation of the Os (II) by the carboxylate group, compound $\mathbf{1}$ was converted to the methylester derivative $\mathbf{2}$. Complex $\mathbf{4}$ was obtained following an adapted literature procedure. ${ }^{20}$ The hydrolysis of the ester ${ }^{24}$ gave complex $\mathbf{5}$, suitable for coupling to a layer of amines on the electrode surface.

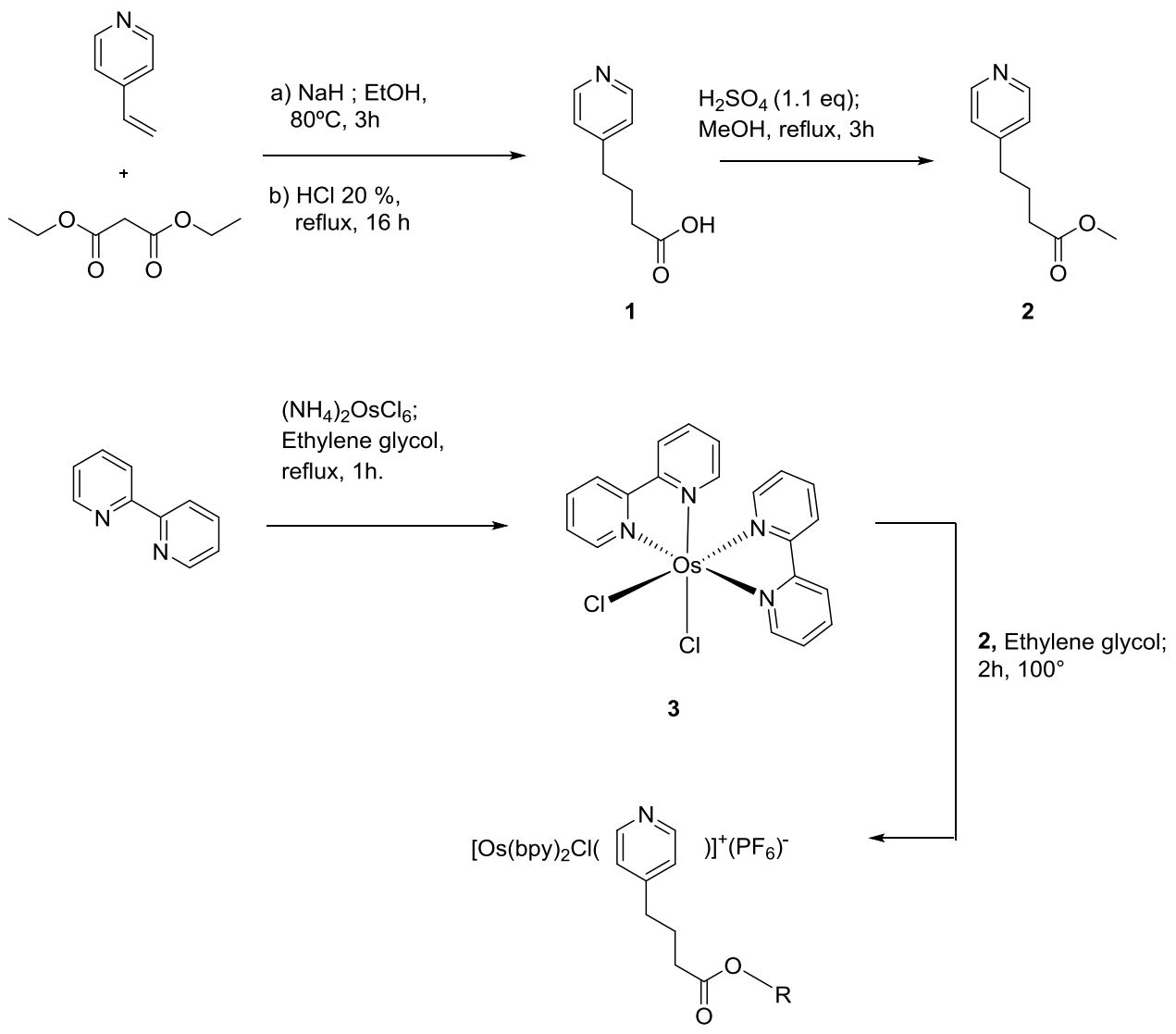

$\mathrm{KOH} 2 \mathrm{~N}, \mathrm{MeOH} / \mathrm{H}_{2} \mathrm{O}$

reflux, $3 \mathrm{~h}$.

4: $\mathrm{R}=\mathrm{CH}_{3}$

5: $\mathrm{R}=\mathrm{H}$

Scheme 1 Synthesis of bis-(2,2'-bipyridyl)(4-(pyridin-4-yl)butanoic acid)chloride osmium (II) hexafluophosphate (5) 
The osmium bipyridyl complex $\mathbf{5}$ was fully characterised and recrystallised by slow evaporation from a $\mathrm{DCM} / \mathrm{MeOH}$ solution. The structure of the complex was confirmed by X-Ray diffraction. The coordinates of the atoms, determined through the crystal structure of complex $\mathbf{5}$, were transferred to the software Gaussian 9.0. Gaussian 9.0 analysed the crystallographic coordinates, using the DFT hybrid method B3LYP with the standard 6-31G basis set for the organic components of the complex while the basis set given for osmium was LanL2DZ, to create a computational model of the complex, where its dimensions could be measured: ${ }^{25}$ considering the pyridyl complex as a sphere characterised by diameter $11.5 \AA$, with a chain giving the overall length of the complex as $16.4 \AA$ (Figure $\mathbf{1}$ ).
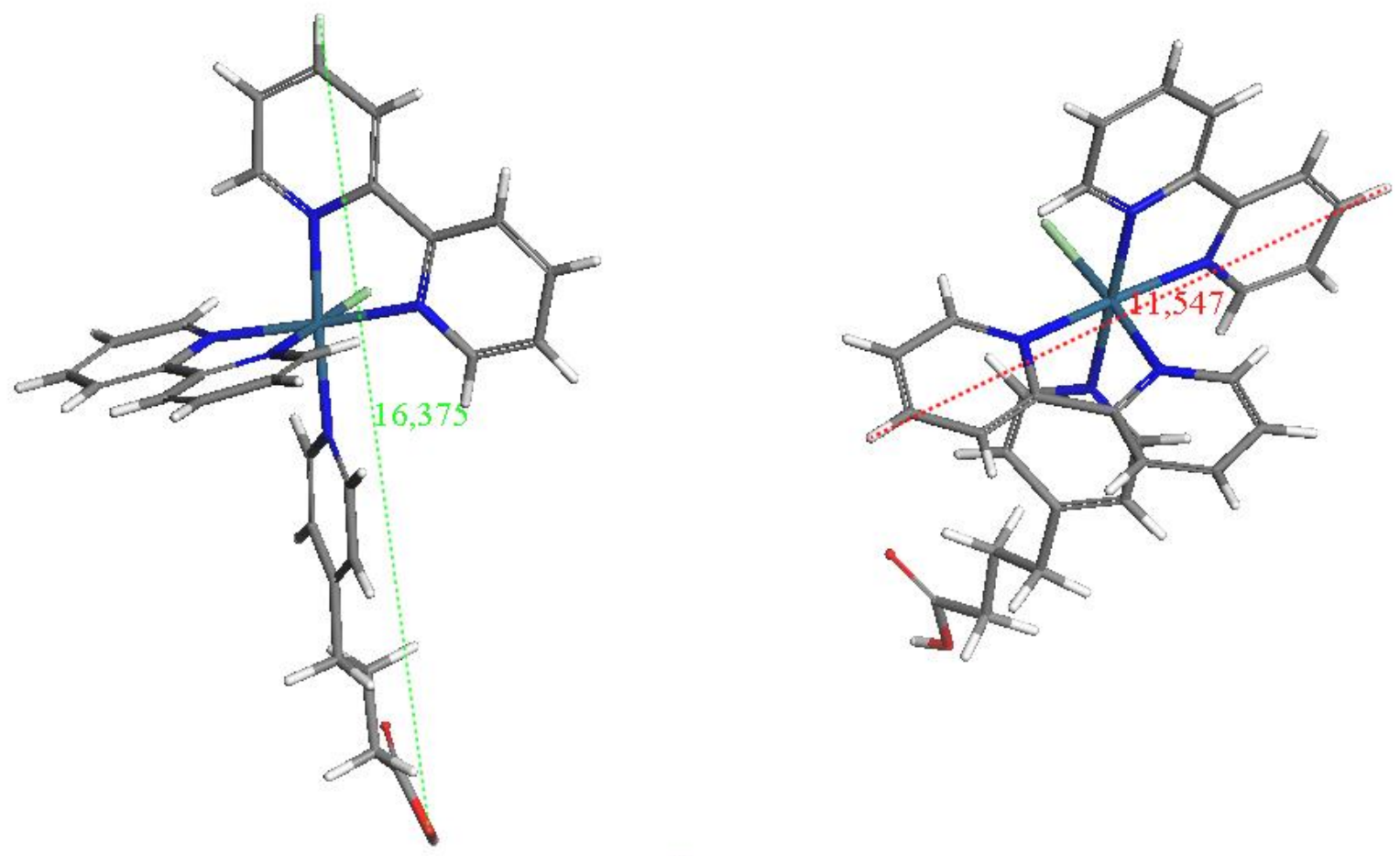

Figure 1 Computational model of complex 5 obtained by analysing the crystallographic coordinates with Gaussian 9.0

These measurements allowed us to calculate the theoretical surface coverage of a monolayer of complex 5. Three assumptions were made: the monolayer of linkers on the GC surface is tightly packed; the amines have no mobility; no repulsion occurs between positively charged osmium complexes. In these conditions two limit situations could occur at the electrode surface: a) the molecules are standing perpendicular to the surface and can be approximated to circles with radius $\mathrm{r}_{1}=$ $5.75 \AA$; b) since complex 5 is attached to the linker through a 4 carbon chain, the molecules could bend 
toward the electrode surface and with free rotation could sweep out an average area corresponding to a circle with radius $r_{2}=16.5 \AA$. In both situations the approximated circles were assumed to organise according to the hexagonal close-packing model, since it represents the densest form of organisation of molecules in a monolayer. ${ }^{26}$ According to this model the area occupied by each molecule can be calculated using the formula:

$$
A=2 \sqrt{3} r^{2}
$$

The surface coverage values for a monolayer of complex 5, calculated knowing the surface area of the

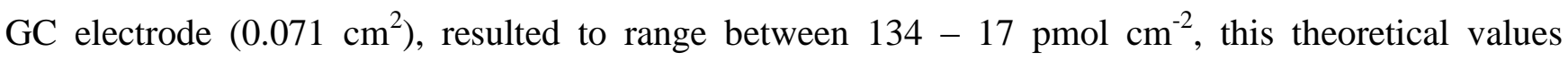
represent a reference to explain the behaviour of complex 5 at the surface.

\section{Mixed monolayers: diamine linker/monoamine}

To investigate the possibility of forming mixed layers at the electrode surface by amine oxidation, a model experiment (Scheme 2) was carried out using a mixture of a linker, mono-N-Boc-1,6hexanediamine (HDA-Boc), and 1-butylamine (1-BA). $20 \mathrm{mM}$ solutions in $\mathrm{CH}_{3} \mathrm{CN}$, with $0.1 \mathrm{M}$ TBATFB as supporting electrolyte, were prepared, containing the two amines in different ratios, starting from $100 \%$ HDA-Boc and gradually increasing the fraction of 1-BA up to 99.9\%. Given the different electrooxidation potential of the components, the grafting of the mixed layer on the GC electrodes was performed by chronoamperometry, holding the potential of the working electrode at 2.1 $\mathrm{V}$ to try to minimise the effects of the different rates of formation of the radical cations of the two amines. Removal of the Boc protecting group under acidic conditions yielded free amino groups available for coupling with complex $\mathbf{5}$ under solid phase synthesis conditions, Scheme 2.

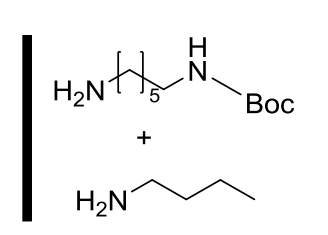

a) $2.1 \mathrm{~V}, \mathrm{CH}_{3} \mathrm{CN}$

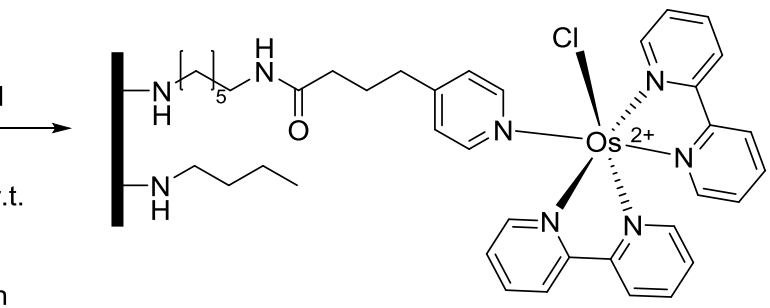

b) $\mathrm{HCl} 4 \mathrm{M}$, dioxane, $1 \mathrm{~h}$, r.t.

c) 5, EDC, NHS, DMF, r.t., $16 \mathrm{~h}$

Scheme 2 Synthetic steps for the covalent modification of the electrode surface with complex $\mathbf{5}$

Figure 2 presents the results obtained: the cyclic voltammograms (CV) show the redox peaks for the $\mathrm{Os}^{2+/ 3+}$ covalently bound to the surface at $\sim 0.25 \mathrm{~V}$ vs. SCE. The decrease in surface coverage $(I)$ of the osmium complex in relation to the fraction of HDA-Boc in the starting solutions is represented in the barplot. The coverages of osmium complex were determined by integration of 
the charge after background subtration (see SI for details) and were corrected by the corresponding controls obtained by dipping the amine modified electrodes in a solution of complex 5 in DMF with no coupling agents added.
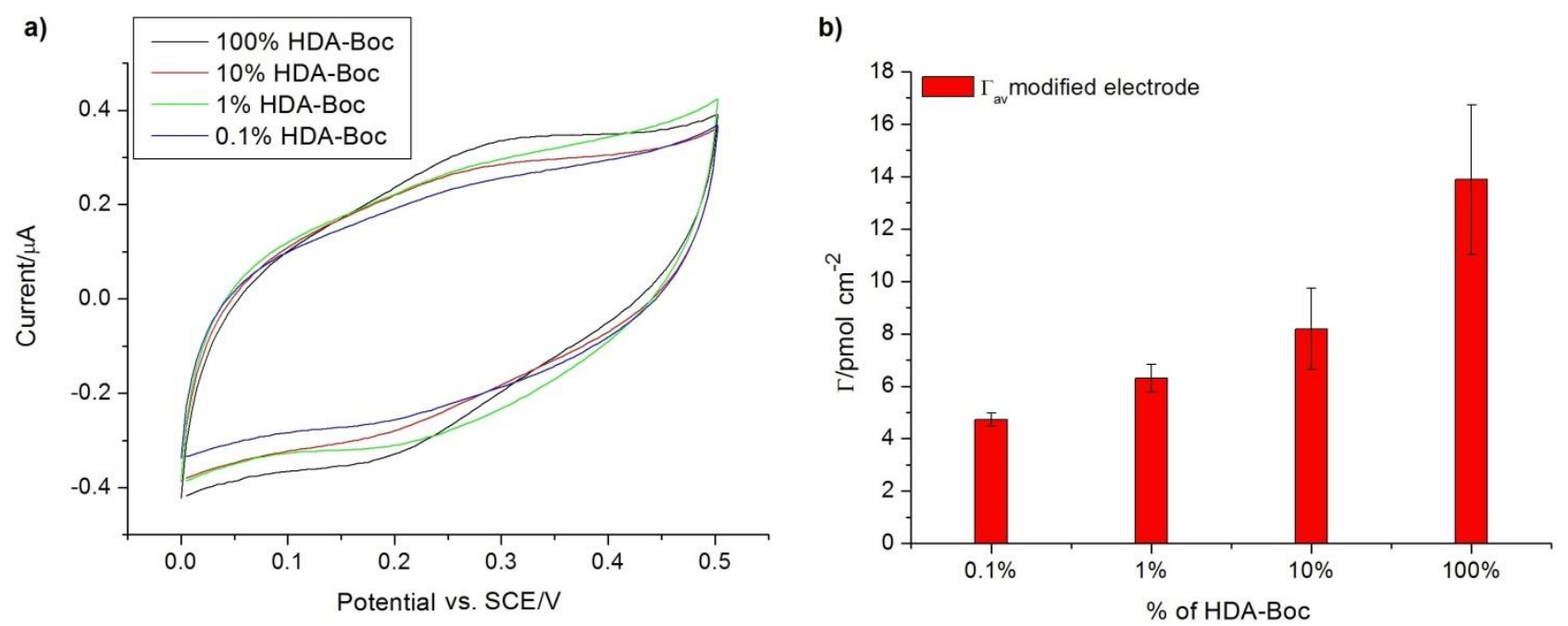

Figure 2 a) Comparison of the CVs recorded in $0.1 \mathrm{M}$ PBS solution pH 7 vs. SCE, electrode area $0.071 \mathrm{~cm}^{2}$, at $50 \mathrm{mV} \mathrm{s} \mathrm{s}^{-1} \mathrm{scan}$ rate for different HDA-Boc linker solution ratios. b) Barplot for the variation of $\Gamma$ for complex 5 according to the ratio of HDA-Boc linker in solution. Coverages were calculated by averaging the values obtained for two replicates and subtracting the control value obtained by dipping amine modified electrodes in a $10 \mathrm{mM}$ solution of complex 5 in DMF without coupling reagent.

It is clear from comparison of $\Gamma$ values in Figure $\mathbf{2 b}$ that the coverage of osmium comples varies only 3 -fold for a $10^{3}$-fold variation in concentration of the HDA-linker. Clearly the relative coverages of the two amines on the surface does not follow the relative concentrations of the two amines in solution. This is presumably due to differences in the rates of attachment of the two amines to the GC surface: attachment of HDA-Boc is presumably favoured over 1-BA, hence the unexpectedly high amount of osmium complex even at low percentages of HDA-Boc in solution. ${ }^{27}$ The surface coverages measured for complex 5 fall within the range expected given the size of the complex determined by theoretical calculation with a coverage of $16-18 \mathrm{pmol} \mathrm{cm}^{-2}$ consistent with model $b$ above in which once coupled to the surface the complex hinders subsequent coupling to amines within its radius of gyration. This also explains why a significant amount of immobilised complex 5 is found even at the lowest $(0.1 \%)$ linker ratio in solution. The coverage for a monolayer of HDA, determined by XPS, is reported to be $\Gamma=1 \mathrm{nmol} \mathrm{cm}^{-2}, 3$ this is 50 times bigger than the calculated surface coverage for a monolayer of complex $\mathbf{5}$, and means that even at $10 \%$ HDA on the surface, if uniformly distributed, a full monolayer of complex $\mathbf{5}$ can still be achieved. 


\section{Mixed monolayers: diamine linker/ diamine linker}

The modification of the GC surface with two redox components, requires a step-wise approach: two linkers must be attached to the surface and selectively reacted with the molecules of interest. It is well known from peptide synthesis that the use of orthogonal protecting groups allows the control of the site of elongation of a peptidic chain, by controlling the conditions, basic or acidic,

of removal of the protecting groups. ${ }^{28}$ Considering this, two mono protected diamines were chosen: mono- $N$-Boc-ethylenediamine (EDA-Boc) and mono- $N$-(6-aminohexyl)-2,2,2-trifluoroacetamide (HDA-tfa): the Boc group can be easily removed in acidic conditions, while tfa requires mild basic conditions. To test this approach initially, grafting of the two linkers to form a monolayer was performed by chronoamperometry from $20 \mathrm{mM}$ solutions of the two components in different ratios in $\mathrm{CH}_{3} \mathrm{CN}$, starting from $100 \%$ of HDA-tfa and increasing the fraction of EDA-Boc up to $99 \%$. After the removal of tfa, leaving the EDA-Boc unaltered, complex 5 was coupled to the free amine groups on the HDA linkers CVs were then recorded and the corresponding surface coverage for the osmium complex calculated. Next the the Boc group was removed, and the free EDA then coupled to anthraquinone-2-carboxylic acid. CVs were recorded for anthraquinone and the surface coverages calculated, see SI for details.

These experiments showed that, although the approach is broadly successful with the coverage of osmium complex increasing and the coverage of anthraquinone decreasing as the ratio of HDA-tfa to EDA-Boc in the initial grafting solution increases there was still a significant amount of anthraquinone attached to the surface even when using 100\% HDA-tfa. This clearly illustrates the problem when trying to attach groups of different steric bulk to the surface. Thus although, as shown in the experiments described above the osmium complex blocks attachment of further osmium complexes to the HDA amine it does not prevent the smaller anthraquinone groups from attaching to the same HDA amines.

To overcome this problem we introduce a further step in which acylation is used to cap any residual HDA amino groups before the removal of the Boc group, Scheme 3. We also found, during the course of these experiments that much more reproducible results could be obtained using glassy carbon electrodes with a meniscus contact to the solution, which eliminates in insulating material around the GC rod, rather than conventional GC electrodes potted in glass (see SI). The meniscus contacting method to the solution was therefore used in all of the following studies. 

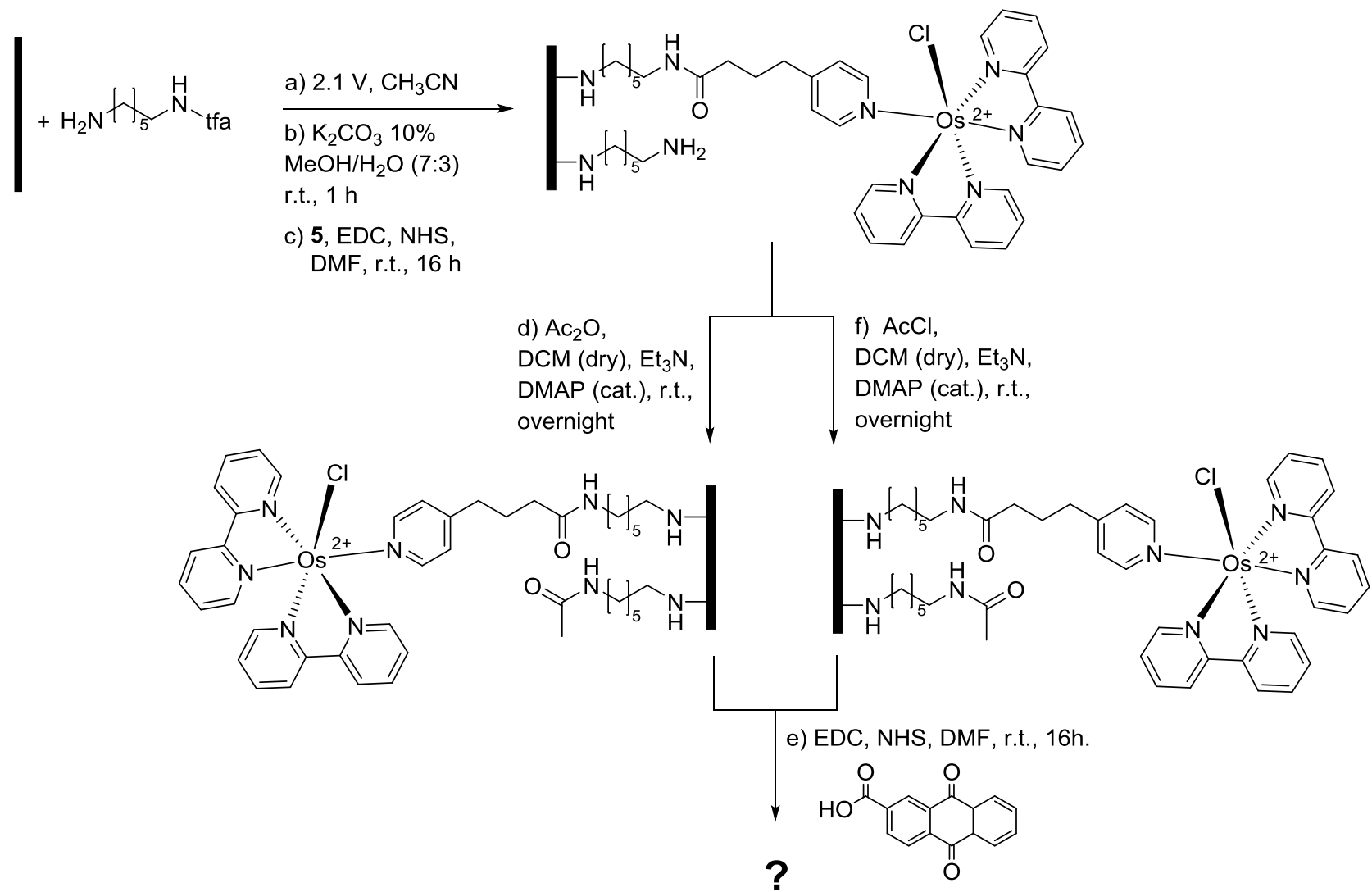

Scheme 3 Synthetic steps for the test of the acylating agents

Acetyl chloride $(\mathrm{AcCl})$ and acetic anhydride $\left(\mathrm{Ac}_{2} \mathrm{O}\right)$ were tested as possible capping reagents. Overall the acylation step led to a significant reduction of anthraquinone at the surface (Figure 3 ) compared to the previous experiments (SI Figure S3), both on modified electrodes, where the anthraquinone surface coverage decreased from $55 \mathrm{pmol} \mathrm{cm} \mathrm{cm}^{-2}$ to $5 \mathrm{pmol} \mathrm{cm}$, and control electrodes, where the surface coverages went from an average of $30 \mathrm{pmol} \mathrm{cm}$-2 to $2 \mathrm{pmol} \mathrm{cm}^{-2}$. This could have meant that the noncovalent attachment of anthraquinone at the surface depended not only on adsorption processes but also on interactions with the residual free amines, such as hydrogen bonding. Acyl chloride gave the lowest values of surface coverage for anthraquinone, so it was chosen as acylating agent. 


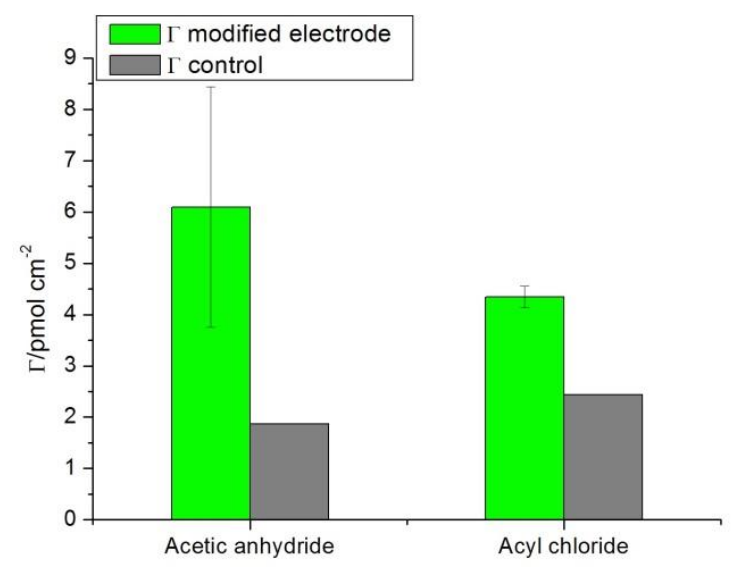

Figure 3 Barplot for the variation of $\Gamma$ of $\mathbf{A Q}$ according to the acylating agent, calculated by averaging the values obtained for two replicates

Once the optimisation of the synthetic steps was completed (see SI), the whole procedure for the creation of the mixed monolayers was repeated (Scheme 4).

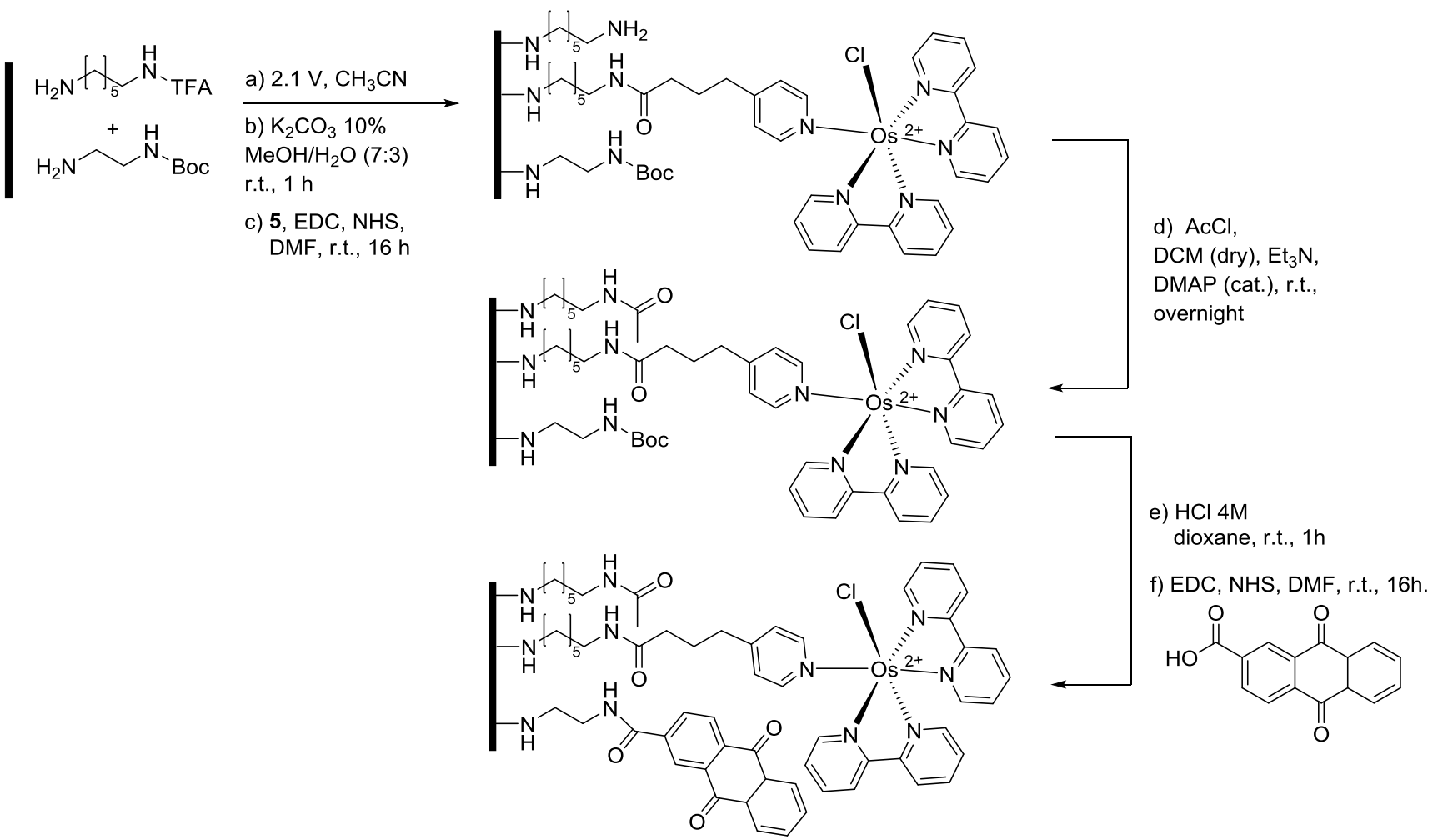

Scheme 4 Optimised synthetic steps for the difunctionalisation of the GC electrodes with complex $\mathbf{5}$ and anthraquinone

Figure 4 summarises the results obtained: the new conditions gave a better control over the variation of surface coverage of the redox species, and the non-covalent attachment of material to the electrode surface was limited to a large extent. The controls for complex 5 obtained by exposing the amine modified electrodes to a neat solution of the complex in DMF, presented no adsorbed material after 20 
$\mathrm{h}$ washing in acetonitrile, while the anthraquinone controls required longer washing time in acetonitrile, $48 \mathrm{~h}$, to achieve a stable signal where no desorption was detected, but still some material remained (typically 2-4 pmol $\mathrm{cm}^{-2}$ ). The values of surface coverage reflected what was expected from previous considerations: at lower concentrations of HDA linker attachment of complex 5 still presents a significant surface coverage. This was due to two factors: HDA-tfa oxidises more easily than EDA-Boc and, although chronoamperometry was applied for the grafting of the amine mixtures to overcome this problem by applying a potential that should generate both amine radicals at the same time, the formation of the HDA radical is probably favoured kinetically, so its concentration at the surface will always be higher than that in solution. Moreover the bulkiness of complex 5 limits the range of $\Gamma$ values achievable as explained above. Anthraquinone shows a much more significant variation consistent with our original expectations and suggesting that the acylation step was successful and necessary. The methodology developed proved to be reproducible and reliable, the total surface coverage of the replicates for each amine mixture tested remained constant within the experimental error, showing that the oxidation of mixture of amines presenting orthogonal protecting groups is a good tool for the creation of mixed monolayers.

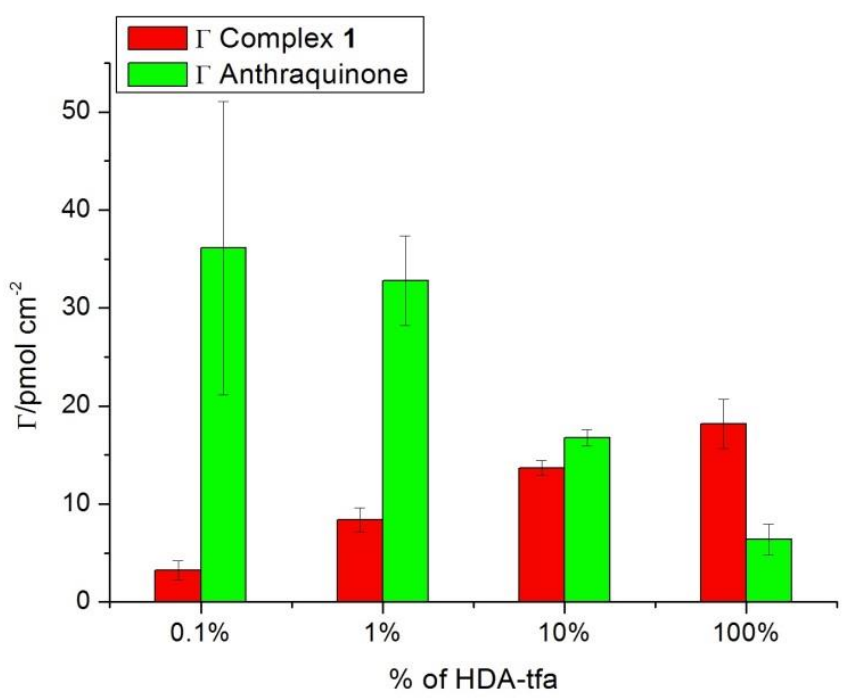

Figure 4 Barplot comparing the variation of $\Gamma$ of complex 5 and anthraquinone according to the ratio of HDA-tfa linker in solution corrected by the corresponding controls. 


\section{Conclusions}

We have described a methodology for the creation of mixed monolayers on GC with a sequential approach, using the electrochemical oxidation of amines and solid phase synthesis techniques. We have shown that it was possible to control the relative amount of two redox probes at the surface by varying the relative amount of the respective amine linkers in solution. The optimisation of the method revealed that many factors have to be considered in order to obtain reproducible and reliable results. The structure of the redox probes has a great impact on the surface coverage values since they can hinder the attachment of similar molecules (osmium complex) or determine non-covalent adsorption (anthraquinone). The setup of the working electrode is an important factor to consider: since the material can adsorb on the sides of the GC rod giving an overestimation of the surface coverage, using the meniscus configuration allowed us to characterise only the desired modified surface. The solid phase synthesis approach applied had to be adapted to the system developed: instead of the classical Boc/F-moc orthogonal protecting groups, the combination Boc/tfa was adopted, in order to avoid interferences by the possible non covalent adsorption at the GC surface of the F-moc group, characterised by a flat aromatic structure. The deprotection conditions were optimised and through the addition of an acylation step, residual free amines left after the first coupling step were capped, procedure routinely applied in solid phase peptide synthesis. The methodology here presented is a simple approach to the controlled creation of mixed monolayers and could be applied to different carbon materials to create much more complex systems for the development of biosensors and biofuel cells.

\section{Experimental}

Materials, experimental procedures for solution and solid phase synthesis and characterisation of the compounds are reported in the Supporting Information.

\section{Acknowledgements}

High resolution mass spectroscopy was carried out by the EPSRC National Mass Spectrometry Service, University of Wales, Swansea. We thank Majid Montevalli for assistance with the X-ray crystal structure of complex 3 and 5 and Dr. Devis Di Tommaso for assistance with the computational model of complex 5 . 


\section{References}

1 a) Barriere, F.; Downard, A.J. J. Solid State Electrochem. 2008, 12, 1231-1244. b) Belanger, D.; Pinson, J. Chem. Soc. Rev. 2011, 40, 3995-4048. c) Murray, R.W. Acc. Chem. Res. 1980, 13, 135141. d) Pinson, J.; Podvorica, F. Chem. Soc. Rev. 2005, 34, 429-439.

2 a) Adenier, A.; Chehimi, M.M.; Gallardo, I.; Pinson, J.; Vilà, N. Langmuir, 2004, 20, 8243. b) Deinhamme, R.S.; Ho, M.; Anderegg, J.W.; Porter, M.D. Langmuir, 1994, 10, 1306.

3 a) Chretien, J.-M.; Ghanem, M.A.; Bartlett, P.N.; Kilburn, J.D. Chem. Eur. J. 2008, 14, 2548-2556.

b) Chretien, J.-M.; Ghanem, M.A.; Bartlett, P.N.; Kilburn, J.D. Chem. Eur. J. 2009, 15, 1192811936. c) Ghanem, M.A.; Chretien, J.-M.; Pinczewska, A.; Kilburn, J.D.; Bartlett, P.N. J. Mater. Chem. 2008, 18, 4917-4927.

4 a) Downard, A.J.; D.J. Garrett, E.S.Q. Tan, Langmuir 2006, 22, 10739-10746. b) Hasobe, T.; Imahori, H.; Kamat, P.V.; Ahn, T.K.; Kim, S.K.; Kim, D.; Fujimoto, A.; Hirakawa, T.; Fukuzumi, S. J. Am. Chem. Soc. 2005, 127, 1216-1228. c) Santos, L.; Mattiuzzi, A.; Jabin, I.; Vandencasteele, N.; Reniers, F.; Reinaud, O.; Hapiot, P.; Lhenry, S.; Leroux, Y.; Lagrost, C. J. Phys. Chem. C 2014, 118, 15919-15928.

5 Unruh, D.A.; Mauldin, C.; Pastine, S.J.; Rolandi, M.; Frechet, J.M.J. J. Am. Chem. Soc. 2010, 132, 6890-6891.

6 Chen, Y.; Yang, C.; Wang, F.-B. Electrochim. Acta 2010, 55, 3951-3956.

7 Imabayashi, S.; Hobara, D.; Kakiuchi, T. Langmuir 1997, 13, 4502-4504.

8 González-Granados, Z.; Sánchez-Obrero, G.; Madueño, R.; Sevilla, J.M.; Blázquez, M.; Pineda, T. J. Phys. Chem. C 2013, 117, 24307-24316.

9 a) Bain, C.D.; Evall, J.; Whitesides, G.M. J. Am. Chem. Soc. 1989, 111, 7155-7164. b) Bain, C.D.; Evall, J.; Whitesides, G.M. J. Am. Chem. Soc. 1989, 111, 7164-7175.

10 a) Darwish, N.; Eggers, P.K.; Da Silva, P.; Zhang, Y.; Tong, Y.; Ye, S.; Gooding, J.J.; PaddonRow, M.N. Chem. Eur. J. 2012, 18, 283-292. b) Darwish, N.; Paddon-Row, M.N.; Gooding, J.J. Acc. Chem. Res. 2014, 47, 385-395. c) Eggers, P.K.; Darwish, N.; Paddon-Row, M.N.; Gooding, J.J. J. Am. Chem. Soc. 2012, 134, 7539-7544. d) Darwish, N.; Eggers, P.K.; Ciampi, S.; Tong, Y.; Ye, S.; Paddon-Row, M.N.; Gooding, J.J. J. Am. Chem. Soc. 2012, 134, 18401-18409.

11 Jeong, S.P.; Lee, B.S.; Kang, S.M.; Ko, S.; Choi, I.S.; Lee, J.K. Chem. Comm. 2014, 50, 52915293.

12 Liu, G.; Liu, J.; Bocking, T.; Eggers, P.K.; Gooding, J.J. Chem. Phys. 2005, 319, 136-146. 
13 Liu, G.; Chockalingham, M.; Khor, S.M.; Gui, A.L.; Gooding, J.J. Electroanalysis 2010, 22, 918926.

14 Liu, G.; Gooding, J.J. Langmuir 2006, 22, 7421-7430.

15 Liu, G.; Paddon-Row, M.N.; Gooding, J.J. Electrochem. Comm. 2007, 9, 2218-2223.

16 Liu, G.; Paddon-Row, M.N.; Gooding, J.J. Chem. Comm. 2008, 33, 3870-3872.

17 Lee, L.; Brooksby, P.A.; Leroux, Y.R.; Hapiot, P.; Downard, A.J. Langmuir 2013, 29, 3133-3139.

18 Chretien, J.-M.; Ghanem, M.A.; Bartlett, P.N.; Kilburn, J.D. Chem. Eur. J. 2009, 15, 11928-11936.

19 Wright, E.J.; Sosna, M.; Bloodworth, S.; Kilburn, J.D.; Bartlett, P.N. Chem. Eur. J. 2014, 20, $5550-5554$.

20 a) Kober, E.M.; Caspar, J.V.; Sullivan, B.P.; Meyer, T.J. Inor. Chem., 1988, 27, 4587-4598. b) Lever, A.B.P. Inor. Chem., 1990, 29, 1271-1285.

21 a) Mao, F.; Mano, N.; Heller, A. J. Am. Chem. Soc., 2003, 125, 4951-4957. b) Poller, S.; Beyl, Y.; Vivekananthan, J.; Guschin D.A., Schuhmann, W. Bioelectrochemistry 2012, 87, 178-184

22 a) Ricci, A.M.; Tognalli, N.; De la Llave, E.; Vericat, C.; Méndez De Leo, L.P.; Williams, F.J.; Scherlis, D.; Salvarezza, R.; Calvo, E.J. Phys. Chem. Chem. Phys. 2011, 13, 5336-5345. b) Garrett, D.J.; Jenkins, P.; Polson, M.I.J.; Leech, D.; Baronian, K.H.R.; Downard, A.J. Electrochimica Acta. 2011, 56, 2213-2220.

23 Menghin, S.; Pertz, H.H.; Kramer, K.; Seifert, R.; Schunack, W.; Elz, S. J. Med. Chem. 2003, 46, 5458-5470.

24 Klein, S.I.; Molino, B.F.; Czekaj, M.; Gardner, C.J.; Chu, V.; Brown, K.; Sabatino, R.D.; Bostwick, J.S.; Kasiewski, C.; Bentley, R.; Windisch, V.; Perrone, M.; Dunwiddie, C.T.; Leadley, R.J. J. Med. Chem. 1998, 41, 2492-2502.

25 Wang, H.; De Yonker, N.J; Gao, H.; Tan, C.; Zhang, X.; Ji, L.; Zhao, C.; Mao, Z.-W. RSC Adv. 2012, 2, 436-446.

26 Vos, J.G.; Forster, R.J.; Keyes, T.E. Interfacial Supramolecular Assemblies, John Wiley \& Sons, 2003.

27 Louault, C.; D’Amours, M.; Belanger, D. Chem. Phys. Chem., 2008, 9, 1164-1170.

28 Dorwald, F. Z Organic Synthesis on Solid Phase ( $2^{\text {nd }}$ Edition.), Wiley-VCH, 2002. 